\title{
Kalledüngung in Budhenjamenjh̨lägen.
}

Bon Dr. Maximirian Serbig, Mitmben, Forfafabemie.

Täufchen nidbt bie İnzetchen, fo ftebt für biejes Jabr, menigitente für gemiffe Gegenden abermals eine Buchenmaft zut erwarten. Erfreulich

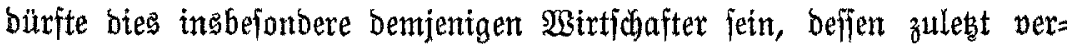
fuchte Butchenverjüngung nibst ben gewounidhten Erfolg batte, befien Wirt: fdaftszalel biefelbe aber von neuem zu veriuchen nötig macht. Unter ben mannigfachen $\mathfrak{u}$ rfacten des Feblgebens möbte id in folgenden Beilen eine beratsgreifen, bie eine genügende allgemeine $\mathfrak{B u ̈ r b i g u n g ~ n o d b ~ n i d f t ~}$ gefunden zu haben idheint: id meine ben Salfmangel in ben oberiten Schidften bes Bodent.

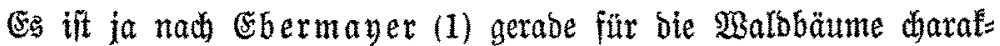

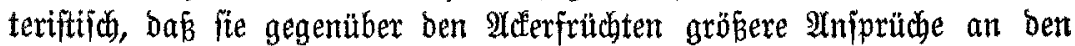
Salfgehalt bes Bobens ftellen; bie Buthe in bejonberen, und nodb mebr bie Buchenjaat ftehen in diejer Sinfït wieber unter ben 2 aldbäumen

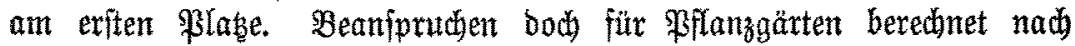
Ungaben $\Re$ a mann's (2) einjäbrige Buduen pro Morgen $52,2=204,4 \mathrm{~kg}$

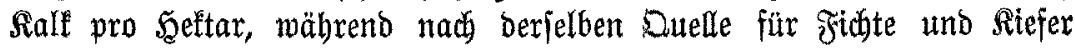
21,8 refp. $19,5 \mathrm{~kg}$ pro Mlorgen ober 85,4 rejp. $76,4 \mathrm{~kg}$ Salf pro Seftar angegeben werben. Sanbmirtibaftlitie Sulturgewädbje aber entbalten nad) Ebermayer (3) in einer Mittelernte pro Şettar

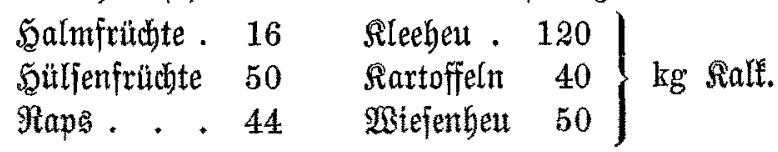

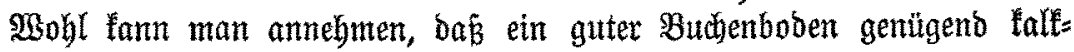
baltig ift; immerbin mag bieje 2 Innahme für bie tieferen Sdjibten bes Bobens ficherer fein, als für bie oberflächlichiten; bie Sitteratur (4) ermähnt Salffelien, bie fith auf 2-3' Tiefe völig an Ralf verarmt zeigten. Wat aber bas Reimbett nidit an biejem, zur Ernährung umum= gänglich notwenoigem, Stoffe bisponiblen Borrat, fo vermögen unter

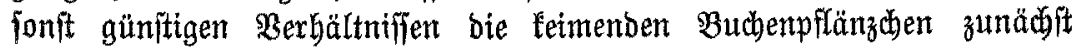
trobbem ein freubiges $\mathfrak{B a c h}$ tum zu zeigen, boch wiro bies nur jolange anhalten, bie bie im Samen mitgebradten Rabtrtoffe fonjumiert finb,

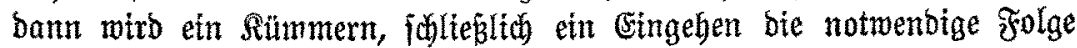

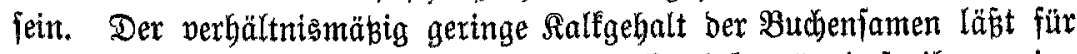
biejelben vermuten ('Berjudbe fino mir nidgt befannt), bap̉ ihnen eine

(1) (2) (3) 2c. Ritteraturangabon fiche Sdituf . 


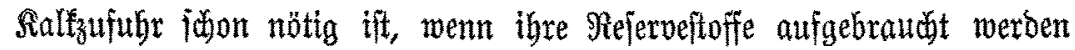
follen.

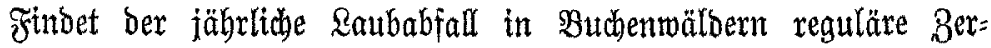
ję̧ung, leibet ber \$oben nidht unter \&aubverwebungen, itbermä̈itger Etreuentrahme $x$., tritt nach bem eventuellen Borbereitungahieb eine ge= funbe Bobenbegrünung ein, bann if anzunebmen, baj ber Boben ge=

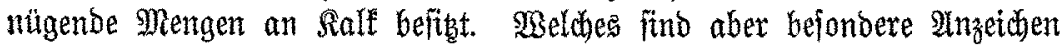
für Ralfnangel? Wenn ber Boben eine ftarf faure Realtion zeigt, ihm

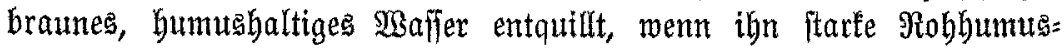

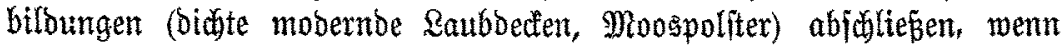
in Bemegung beftnoliche eifenverbinbungen zur abjetetbung won Braun= eifen in Sörnern und Streifen, als eifenverbärteter Sand und Eifenort=

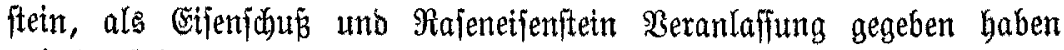
und Drtftein in $\mathfrak{u}$ ntergambe auftritt, enblid) wenn eine "fautre" Siege= tation (F̧eidelbeere, Freifelbere, Seibe, Farn 2c.) bie vorberribende ift.

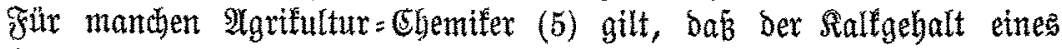
Rultur=Bobens nibt unter 0,2 pet. Itegen follte, (bod) fann fold einer

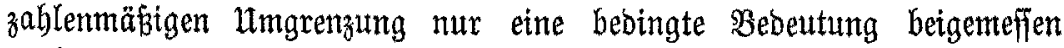
werben.

Beldhe Birfungen läpt eine Raltoüngung im Speziellen erwarten? Der bireften Wirfung als abjolut notwenbiges Räbrmaterial ift bereits

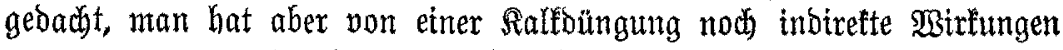
zu erwarten, vermöge berer er fabn burch feine IInwejentyeit als joldhe (abgejeben alfo von leiner Mähritoffetgenichaft) bäufig in Boben eine Extragsifteigerung bewirft, in benen offenbar fein Mangel an biejem Mineral berridht. $3 \mathfrak{u}$ biefen indireften $M$ irfungsweifen gebört in erfter

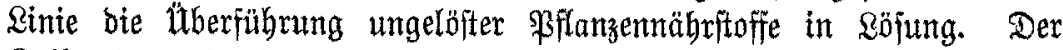
Salf tritt 3.3 . in bie im Boden worbandenen mafierbaltigen Silifate ein tno verbrängt baraus bie alfalien: Sali und Ratron, bie baburd) ben Pflanzentwurzeln leiğter verfügbar merben. ${ }^{1}$ ) Durch jene Mechjel= wirfung aber befommen die Bobenlöjungen eine veränderte Bujammen= jebung uno beipülen nun bie Bodenteile mit Stoffen, bie jonit vielleidft lofalifiert geblieben wären, gelöft aber zu weiteren, für bie Ernäbrung

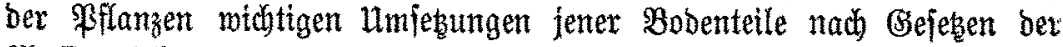
Maffenmirfung 2 . fübren fönnen. Doch nidbt allein rein anorganifid gebunbene Mährmineralien fest Salt um, auch organtiobe, wie bie im Sumus inveftierten $\mathfrak{B e r b i n t o u n g e n ; ~ i n b e m ~ e r ~ h i e r ~ b i e ~ s e b e n s t h a ̈ t i g f e i t ~}$

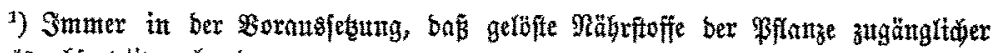
finb, als abjorptio gebunbene. 
gewiffer nieberer Drganismen begünftigt, befhleutnigt er bie Berjegutng ber vorbandenen Şumugjubitanzen. Rohhumusmaffen, bie megen ibrer

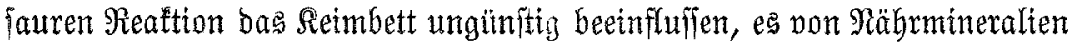

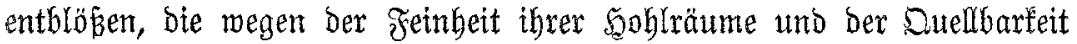

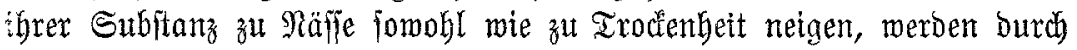
Salf neutralifitert und rafderer Berfebung entgegengefübrt. Die bie Bege=

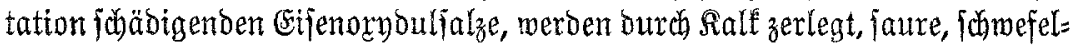
faure Salze abgeftumpft, Eifentitriol wiro in Gyps und sifenoryoulfydorat übergeführt, Das fich an ber Ruft leibt zu Eifenorvohyorat orndoert und als

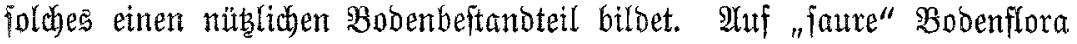
(Šeide, Sreifelbeare, Binjen zc.) wirft falfhaltiges $\mathfrak{B a f i j e r}$ wie ein Bift uno verurjacht bexen 2lbjterben. Strenge, febr binbige Thon= uno Rehnböben

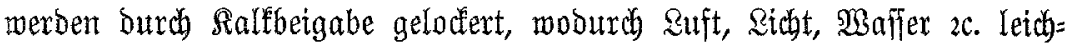
teren 3utritt erbalten; letchte, fambige Böben aber merben (befonders Durch Thonmergel) binbiger gemacht, weniger burdjläffig utto baburch

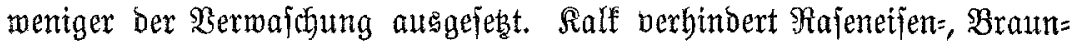
eifen= uno Drtfteinbildung, wo aber jolche ftatthat, wirb Salt a. a. ale Saeilmittel empfohlen. Fiad Stahl (6) jollen Pflanzenzellen, bie Ralf=

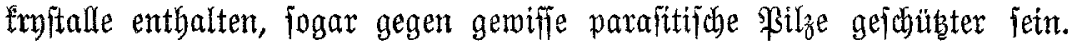

Die Wirfung Des Ralles auf feimende Samen illuftriert jebr gut ein $\mathfrak{B e r j u d}$ von \&iebenberg (7), nadh bemfelben ergaben Keimende

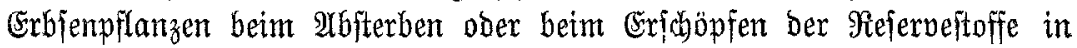
Den Reimblättern bie nachfolgenden Stengel= uno $\mathfrak{B}$ Burzellängen:

\begin{tabular}{|c|c|c|c|c|c|c|}
\hline & $\begin{array}{c}5 \\
\text { größzte } \\
\mathrm{cm}\end{array}$ & $\begin{array}{l}\text { tengellat } \\
\mathrm{cm}\end{array}$ & $\begin{array}{l}\text { nge } \\
\text { Mittel } \\
\mathrm{cm}\end{array}$ & $\begin{array}{r}\mathfrak{B} \mathfrak{1} \\
\text { gröñte } \\
\mathrm{cm}\end{array}$ & $\begin{array}{l}\text { itrzellân } \\
\mathrm{cm}\end{array}$ & $\begin{array}{l}\text { Ige } \\
\text { Mittel } \\
\mathrm{cm}\end{array}$ \\
\hline \multicolumn{7}{|l|}{ a) Waffer unb Röfungett in $\mathfrak{k a f f e r}$} \\
\hline 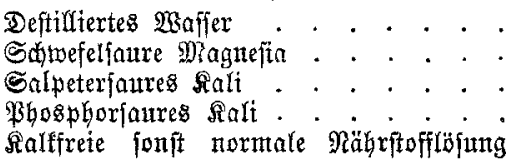 & $\begin{array}{l}9,- \\
4,50 \\
5,50 \\
6,50\end{array}$ & $\begin{array}{l}4,- \\
2,- \\
3,- \\
3,-\end{array}$ & $\begin{array}{l}5,60 \\
3,45 \\
4,07 \\
4,30\end{array}$ & $\begin{array}{l}9,- \\
3,70 \\
6,- \\
6,-\end{array}$ & $\begin{array}{l}3,- \\
2,50 \\
3,- \\
4,-\end{array}$ & $\begin{array}{l}7,07 \\
2,95 \\
3,64 \\
5,62\end{array}$ \\
\hline $\begin{array}{c}\text { nad Rnop } \cdot \cdot \cdot \cdot \cdot \cdot \cdot \cdot \\
\text { b) Ralfbaltige \&öfungen }\end{array}$ & $5,-$ & $2,-$ & 3,56 & $5,-$ & $3,-$ & 3,68 \\
\hline 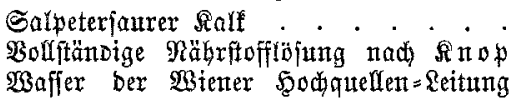 & $\begin{array}{l}40,- \\
34,- \\
40,-\end{array}$ & $\begin{array}{l}20,- \\
25,- \\
29,-\end{array}$ & $\begin{array}{l}29,33 \\
27,42 \\
34,87\end{array}$ & $\begin{array}{l}22,- \\
31,- \\
28,-\end{array}$ & $\begin{array}{l}14,- \\
19,- \\
15,-\end{array}$ & $\begin{array}{l}18,50 \\
25,42 \\
19,12\end{array}$ \\
\hline
\end{tabular}

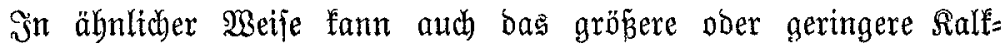
bedürjnis eines Malobobens an bem Madhstum ber Fffanzen Durd ben Topfperiuct) gemefijen werben. Man ftelle zu biejem Zmed zwei $\mathfrak{B}$ er= 
juthereihen an, eine mit, eine ohne Betgabe von $\Re a l f$. Bon lekterem

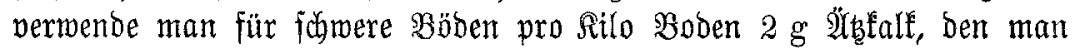
zu einem trodfenen Pulver löjht, innig untermijht, bann aber bas (Be= menge erit einige $\mathfrak{T} a g e$ fich felbft überläbt, bevor man mit bem (Einjäen beginnt. Für leidfte Böben benutbe man foblenjauren fall in Form von

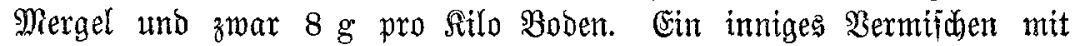
Dem Mutterbodent ift hter ebenjo zu beachter, nur Lann bas Einjäen ber betreffenden Serjucbsobjefte bier jofort erfolgen, nadbdem man ben Boben

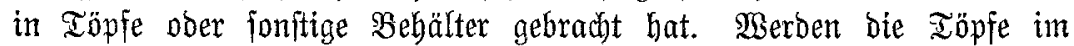

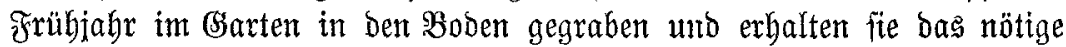
Waffer, fo fann über bas Salfbedürfnis eines Bobens wobl ein gewifier 2 nnhalt gemonmen werben; immerbin finto bas nur gärtnerifde \$orver= furbe, melche benjenigen bie auf freier $\mathfrak{3 a}$ aldflädhe vorgenommen werben, nicht völlig vergleidhbar fint.

\section{Welche Waterialten fommen für eine Kalfoüngung in frage?}

Sn erfter sinie gebrannter Salf und fohlenjaurer Salf, leşterex in Form von Mergel ober gemahlenem Ralfftein, in zweiter Rinte gewifje falebaltige 2lbfallitoffe.

\section{a) Grebraunter Sialf (CaO).}

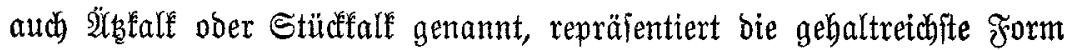
und enthält als Şandelsprodult $90-95$ pet. Salferde ober wirtjamen

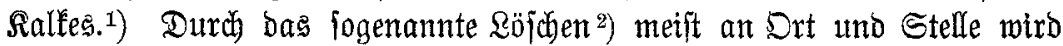
er unter Mafferàufnahme in bie ftreubare Form bes Ralfyydratee ober

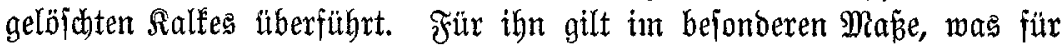

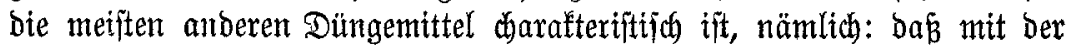

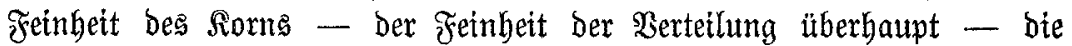

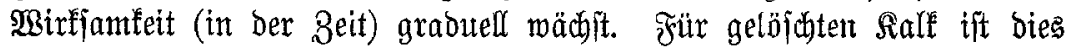
noch von bejonderer Bedeutung, weil er burdh eine ausgejprodbene Reigung fich mit ber Roblenjäure in \&uft und Boben za verbinben, baburdb

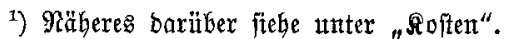

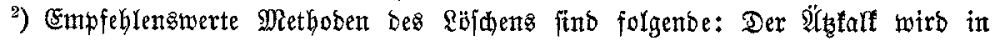
fleinen Saufen liber bie entfpredenbe Flädhe verbreitet thb gleid barauf intt $1 / 3$ feites

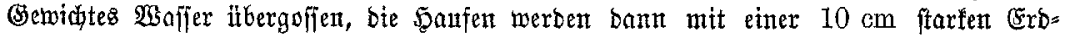

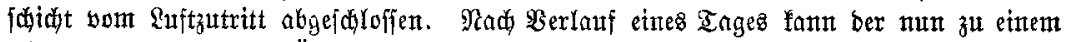

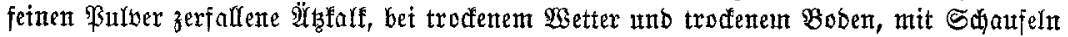

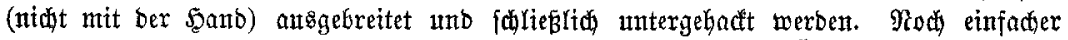
ift, ntan tautht bie in eittem Rorbe befinblidie beftimmte Menge ä̈bfalf in eine Ionne

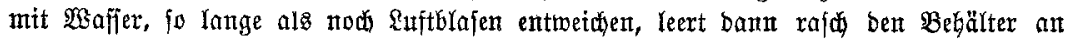
ber vorgejebenen Stelle, bectt mit Erbe ab unt berf̧ăhrt woie oben. 
wieber in kohlenfauren Ralf übergeführt wirb, biejelbe Mobiftlation, bie ihm vor bem Brennen eigen war, bie fich weniger wirkungefräftig erweift uno langfamer umiest. Gerabe bieje fräftige und rajde $\mathfrak{B}$ Binfung bes

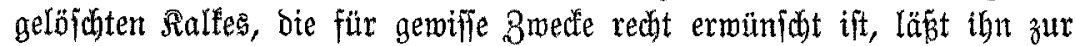

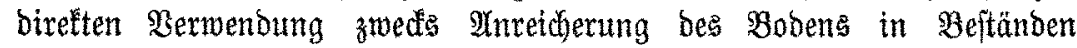

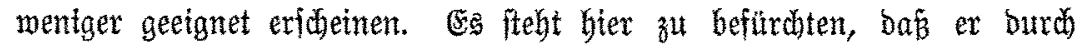
zu befdhleunigte Berfebung bes Satmus ben Boben von bemfelben ent=

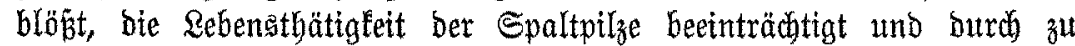

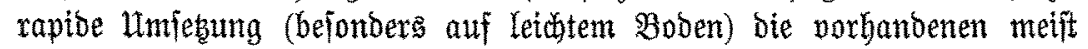

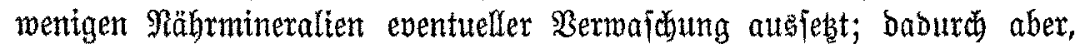
unb verftärft burch gröpere Bobendurdfläjïtgheit, farm ber im Mafier leidyt

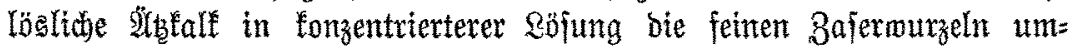
fpülen, fie anäken unb eventuell zum 2lbjterben bringen. Es ift baher vor bex 2lnwendung von gelöfhtem Rall und gebranntem Ralf in Stüflen zur Änreicherung in Bejtänoen zu warnen, folange, bis Berjuche, die hier eingeleitet find, feine Wirkung barzuthun, ein Urteil uber feine Braudbarteit ermöglichen. (Exheifden wirtidgaftlidge (Srünoe trobem bie

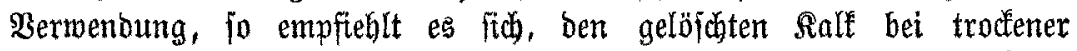
Witterung auf bem trodenen Boden auggeftreut einige Beit liegen zut lafjen uno bann erft eitzutbaden. Dieje Mantpulation fumpft bie heftige

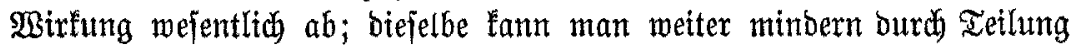
Der aufzubringenden Duanten, ober beren vorherige Rompoftierung. $\mathbb{Z}^{\mathfrak{t}}$ crwähnet wären bier nod) bie fogenanten (orau= ober bolomitithen

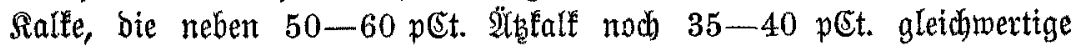
Magnefia enthalten, nur etwas wentger intenitn, aber um jo nadyaltiger wirfen (8). $\quad \mathfrak{D b}$ und imwieweit biejelben zur Berwendung in altbeftänben vorteilfaft itno, wäre um io intereffanter zu ermitteln, als $\mathfrak{B} \in \mathbf{e b e r}$ (9) bas Solz ber Buche nach einem Samenjahr nicht allein an Eimeţ́ fondern

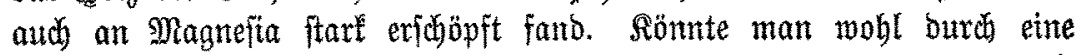
Magneftazafubr auf bie Samenbiloung etmwirten? Wäbrent im Solz

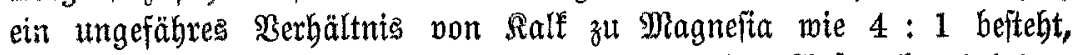
ftellt fith basjelbe in ben Samen mie $2: 1$. Der 2 nnjprudh 1 jähriger

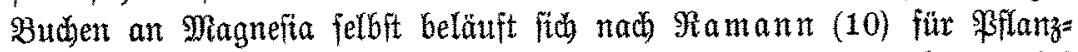
gärten beredinet auf $9,9 \mathrm{~kg}$ pro Morgen $=38,77 \mathrm{~kg}$ pro 5 Seftar, wobet zu berücfirdytigen ît, bas Magnefia ben Ralf relativ zu erfeten vermag (11).

\section{b) Sofitemiaurex $\mathfrak{R a f t}\left(\mathrm{Ca} \mathrm{CO}_{3}\right)$}

in Form won Mergel ober gemablenem (ungebrannten) Ralkftein bürfte für Buchenjamenichläge biejenige Mobififation jein, die als Mittel für unjern Broed unbedenflith empiohlen werden fam. 


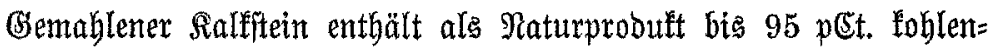
fauren Raltes, bas entiprtat ca. 53 pet. wirffamer Rallerbe, ${ }^{1}$ ) währeno

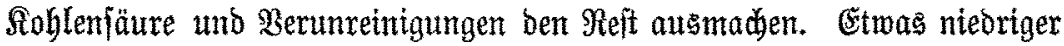
im Gehalte ftehende fohleniaure Ralfe bezeidhnet man im allgemeinen als "Mergel", boch fommen unter biejer Bezeidjuntg Sorten in מ̆anoel vor, bie beftem, gemablenen, fohlenfaurem ßalfe in Gehalt und Feinbeit ber Siebung volftändig ebenbürtig fino. Die im norbbeutiden Fladilanbe weit verbreiteten biluvialen Mergelablagerungen befteben zumeit aus Gemengteilen von lohlenfaurem Salk, Ihon unb Sand, je nabbem ber eine ober andere Beftandteil überwiegt, unterideibet man $\mathfrak{\text { Salt }}=$, Ihon= uno Sandmergel. Der Bebalt berjelben an Foblenfaurem Ralf it in ber $\Re$ Regel lein boher, 30 prozentig gilt er jibon als ein guter, 50 prozentig nennt man ifn nach Rönig (12) "fett", barunter "mager". $21 m$ vor= teilhajteften hat fich erwiejen, auf leidotem Boben Ihonmergel, auf johwerem Sanomergel zu benuben. Raffe, falte Rebmböben werben burdh Sandmergel lodferer und trodener, loje Sandböben burd Thoumergel fefter, bindiger uno bamit mafferbaltender. Bon ber Sigenidaft biejer

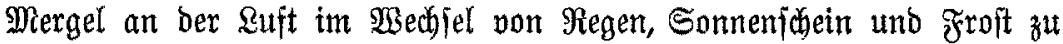

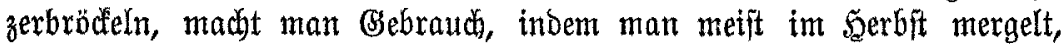

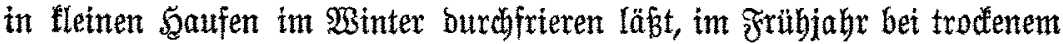
Better bie pulverige Mafje auseinanber ftreut unb bann in innige, gleid: mäpige Bermengung mit bem Mutterboden bringt. Durch längeres Sagern an ber \&ujt werben mandhe ichädiche Bejtandteile, wie Sdywefel= eifen, wenn tie bei \&uftabichlup entitanden fein follten, infolge Sauter ftoffaufnabme zeriebt. Die aus bem Samefel aber fo entitanbene

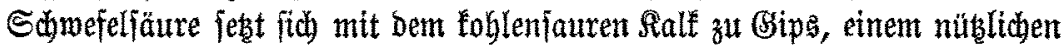
Bobenbeitanbteil, um. Für 90-95 prozentige präparierte $\Re$ alfmergel finto natütlid bie Rebenbejtandteile von geringerer Bedeutung; bieje Mergel fommen meift jo feintërnig in ben Sandel, ba Fribjabx geftreut unb untergebradt werben fönnen, boh bies im Serbit z̆ thun, iff immerbin vorzażtehen.

\section{c) Ralfaide vier Etaublalt}

bilbet ein $\mathfrak{2} b$ fallprobuft ber Salfbrennerei, je länger an ber Suft liegend, um fo mebr fintt ber âdefalfigebalt uno um fo böber fteigt bamit ber= fenige an foblenjaurem Ralf und bte Bertverminberung.

\section{d) (็ngtalf}

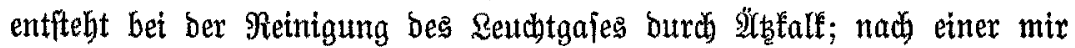
vorliegenden YMalyje (13) enthielten Froben $34-49$ p(t. Falt in Form

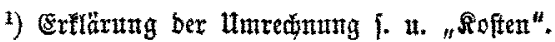


von fobienfaurem Ralk, 2̌lthalf und Gipe neben geringent Mengen von Stidfitoff. Un einen eventuellen Bsehalt an echwefelcalcium uno ithwefelig= faurem Ralfe, bie bie Begetation nadjteilig beeinfluffen, umiöäblici zu machen, ift er längere Beit auggebretiet an ber Suft liegen zu laffen, ehe man inn meiter permentet.

\section{e) Sdieibeidilnum}

ber Zucterfabrifen, ber fid) um fo mehr wirfungsooll ertweift, als er $\mathfrak{u}$ a. neben 20-30 pet. Hoblenjauren Saffes, nod etwa 1 pet. \$hosphor= fäure, 0,3 p@t. Rali unb 0,5 pet. Stidftoff entyält (14). Ma wer (15) fano etwas ntedrigere Merte; bet berartigen Produfften vermögen bie= Felben natilltid nidt fonitante zu jein!

\section{f) Seijenitiederfate.}

Die bei bent früberen Darftellunggverfahren für Seifen verwanbte Golgajose gab dem abfalffalf gemiffe wertwolle Beimengungen von phos= phoriauren Salzen; neuerbings aber, ba man zur Seritellung ber Seife meift Soba verwendet, befteht ber Seifenfiederlalf weientlich atts loblent= jourem Salf neben etwas ît jeweilige Gebalt ift, wie bet allent Ilbfalftoffen, wedbjelno; eine von Drth unteriutedte $\$$ frobe (16) ergab:

$$
\begin{aligned}
& 35,15 \text { pet. lohlenjauren Salk, } \\
& 13,05 \text { "Salfbyorat (= gelöichter Ralf), } \\
& 0,78 \text { " fohlenịoure Magnefia, } \\
& 0,17 \text { " Rodjalz, } \\
& 43,89 \text { " Maffer. }
\end{aligned}
$$

Diejer hohe :affergebalt erböht natürtich bie Untoiten, um fo mehr, als er ein vorheriges Irodinen bes Frobuftes vor ber biretten Bermen= bung nötig maddt.

\section{g) (sips}

befteht aus wafferbaltigem, fadwefelfaurem Sall $\left(\mathrm{CaSO}_{4}+2 \mathrm{H}_{2} \mathrm{O}\right)$, rein

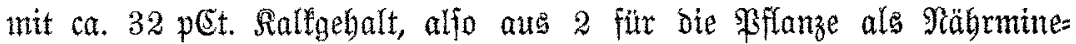
ralien notwenbigen Stoffen. Der Sorteil einer Gipsoüntung beruht jeboch weniger barauf, als auf ber indiretten (bislocierenden) SBirfung, bie thn unter Den Salffalzen bejonders auszeidfnet. Er ift ungebrannt uno gemablen für Düngungszmecte bereits vermenobar. Die Ranowirt= finaft bemukt ibun mit gutem Crfolge in Mengen won $500 \mathrm{~kg}$ pro şeftar

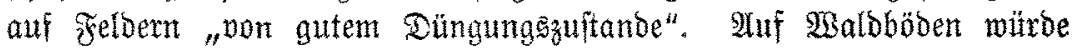
beghalb, iwenigitens vor einer Serwenbung gröperen Stils, ein Frobe=

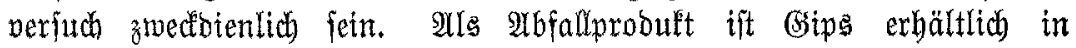
Stearinfabrifen, Rartoffielzadferfabrifen, betm Salimenbetrieb $2 \mathrm{c}$. 
Steinfohlens, Braunfoblen= tuo Torfajden enthalten ebenfo immer medjelnde Mengen von Ralf, neben Rali uno \$hosphorjäure; man ver= wente erjtere befier geftebt, weil gröbere, foblackenartig geidhmolzene Ietle

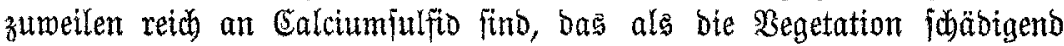
bereits ermähnt wurbe. YUub Strakenitaub mirb, je nach feiner Ger= funft, bejonbers aber wegen feiner fermentativen Bitrfung als mely ober weniger falfhaltiges Düngemittel vermentet.

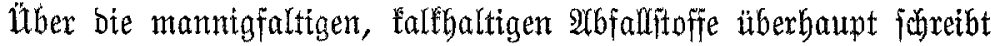
mir ber, um bie Frage ber füntlithen Düngung woblverbiente, hollän=

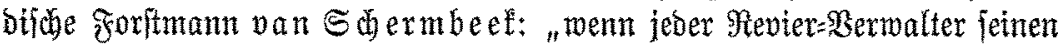

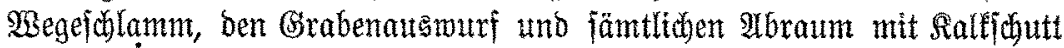
pon Saumerfen, überbaupt mit jebem falfreidjen Material zujammen= bringen wollte auf veridjiedenen Funten, in jedem Belauj, bann fömnte bie Zrage nicht von fo gefährlichen Dimenftonen weroen." Beziebt fich

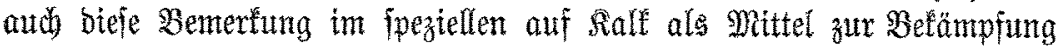
ber Robhumusbiloung, fie ift ebenjo aufe angemeinere anwenbbar; fither finden fith bier und ba fallfaltige 2lbfalftoffe, bie fith für ben $\mathfrak{B a l d}$ mit $\mathfrak{B o r t e i l}$ nerwenden Yiešen, bisher mur letne Beachtung fanden; natürlid barf ber Wirtfigafter babei bas Grempel nicht pergeffen!

\section{Bodenworbereitung.}

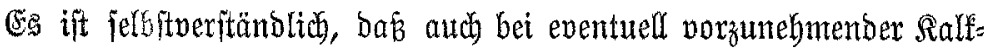
bünung alle b̧ilfşmittel ergriffen werben, berer man fid fonft bebient, unt bem Boben bie nötige "Gahre" zu vexidaffen! Bejentlity fïr ben Erfolg ber Ralfoüngutg ift Wusftreten bei trodfenem Better, auf trodenem Boden und vor allem möglidfit inniges Sermifiben mit bent $\mathfrak{T}_{\text {utter }}$ boden. Man Laffe besgalb die Rollegge vor unb nach bem Streuten bes Ralfes gehen unb hade ein, wo biejelbe nidjt forgjam genug arbeitet refp. arbeiten 弚m.

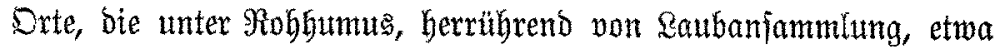

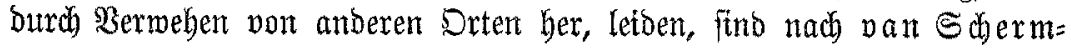
beef (17) worber am beften burd Entfernung des \&aubes unter $\mathfrak{b e =}$ lafjung ber toten Dede zât räumen. Das entfernte Material ift zwed =

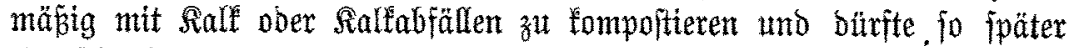
ein Föăbares Düngematerial, ppez̧iell für humusbedürftige Böben $\mathfrak{a b}=$ geben. Snmiemeit burch eine pafiende (Gruppen= ober Streifenverjitngung bem ütbel Künftigen \&aubnermełens vorz̆ubengen ijt, wiro bem lurteil bes Mixtjobafters at belafien fein. 


\section{Welche Quanten find ju verwenden?}

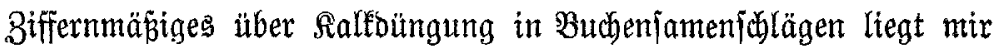
nidyt vor; bie hter eingeleiteten $\mathfrak{B}$ erjuche fint fomparative, es follen zut= näbhft $500-2000 \mathrm{~kg} 90$ prozentigen Mergels uno biejelbe Mienge 90 pro=

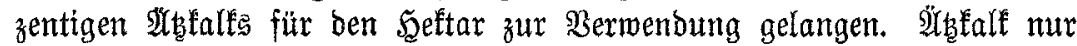
in Beftänben, bie, zum Şiebe beftimmt, etwaigen Sdjaben nidjt anfteigen zu lafjen vermögen $\mathfrak{m} \delta$ mehr $\mathfrak{u m}$ Dasjenige Dutantum $\hat{z}^{\mathfrak{u}}$ ermitteln, meldes auf einmal gegeben bier gerabe noch vertragen miro.

Weldhe Mengen aber felbft 2 Anreicherungen geringen Grabes erforbern,

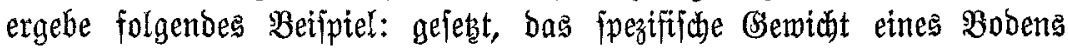

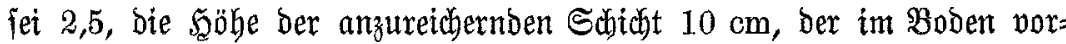

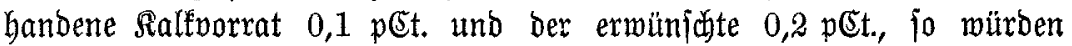
$2500 \mathrm{~kg} 100$ prozentigen $\mathfrak{2}$ (hét Duantum, bas in Form von Mergel auf rein lanbmirtfaftaftich benukten Flächen faft aller Bobenflaffen Serwendung findet. Doch bandelt es ftch Da um unbeitanbene ₹lächen uno erprobte Mengen. Man würde ebenjo

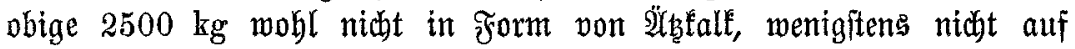
Sanbboden und auf einmal, dem Boben einverletben; es ftände zu be= fürobten, dás wie Salfelb=Ringen (18) bei Mengen won $2000 \mathrm{~kg}$

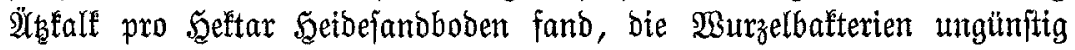
beeinflǘt und bie Erträge geminbert würben.

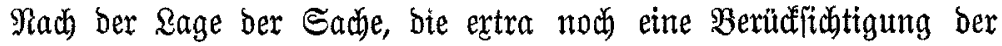

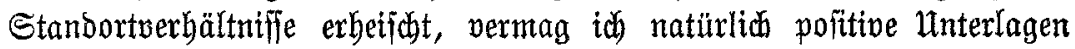
für eventuell anzumenbende Duanten nicht zu geben, überlaffe es vielmebr

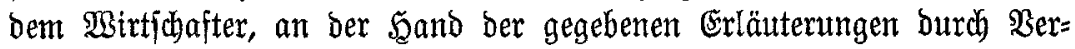
quche fleineren ober größeren Sttis fich ein Utrteil über bas eventuelle

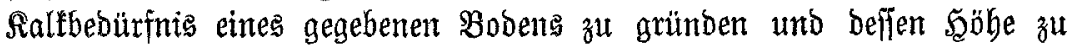
bemefien. Da bei Mergel eine jhäbigenbe Mirfung faum zul befürchten ift, fönnen bejonbers wo berjelbe biflig zat baben ift, felbjt reichlidse Duanten verjuchsmeife gegeben werben, man itufe ab je nachbem 2 Uuf= johlag überbaupt nicht erzielt murde, bald werbuttete, voer baloiges $\mathfrak{B e r}=$ butten wahridbeinlid macht, für leksteren Fan jollen bjer $1000 \mathrm{~kg} 100 \mathrm{pro}=$ entigen fohlenjauren Salfes pro Szeftar in Form eines 90 prozentigen Mergelg feiner Mablung, übergeitreut, \$̧erwentoung finden.

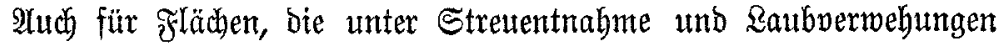

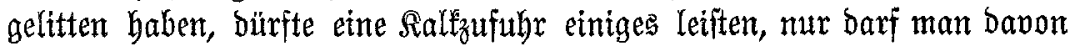
nidbt erwarten, dás fie bas entitandene Minus an Mäbrmineralien wie Sali, \$hosphorjäure, હtidfftoff 2 . vollmertig aufžtbringen vermag; immer= bit witro eit Erfolg fichtbar werben, beutlidber auf sehm= benn auf Sanbböben. 


\section{Kojten.}

Die Rioften ber Ralesüngung werben naturgemäp je nach ber ört=

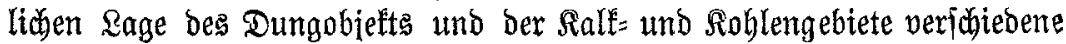
fein. Barieren werben ebenio bie \&öhne. Da bei einer Ralfbüngung bie Roften für bag Material jelbft burch biejenigen, bie für Trangport

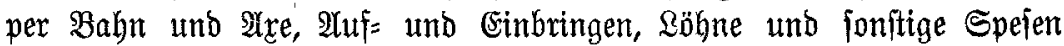
entiteben, meif́t übertroffen werben, ift es zweeflos, auf erftere einzugehen, Sur Ralfullation felbft aber biene folgendes: Der mertoolle Teil ber Rall= büngemittel berubt in ihrem Gehalt an salferbe = Sllbfalf $(\mathrm{CaO})$; dieje Mobififation finbet fich wie bereits erroähnt im gebrannten $\mathfrak{A a l f}$ bis zu 95 pCt., währeno im fohlenfauren Ralf und im Mergel bie Salferbe nod Durch Rohlenjäure $\left(\mathrm{CO}_{2}\right)$ befchwert ift, chentifa) rein würde ihr Gebalt an Ralferbe $56 \mathrm{pCot}$. betragen. Soldhe Sorten fommen natürlich für Dungzwedfe nidht in Betradbt, immerhin werben Mergel angeboten von 90 p〔t. foblenjautren $\operatorname{Ralfe} e$, bag find bei $100 \mathrm{~kg}\left(\frac{90 \times 56}{100}\right)=50,4 \mathrm{~kg}$ Ralferbe.

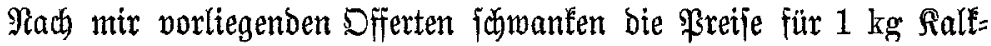
erde zwifden $1 / 2$ und 2 gू. Da, wo ein weiter Irangport nötig ift, wiro bie $\mathfrak{B}$ ermendung hodpprosentiger $\mathfrak{B a r e}$ mehr in Frage fommen. Man faufe nadh Rilo Salferbe unter (Garantie des Gehaltes. ${ }^{1}$ )

Bezug in $\mathfrak{B a g e n l a b u n g e n ~ v e r b i l l i g t ~ n a t u ̈ r l i c ~ b e s e n t e n o , ~ b i s ~ z u m ~}$ 30. 2 pril 1902 verfradbtet überbies bie $\mathfrak{B a h n}$ "Ralfhaltige Düngemittel"

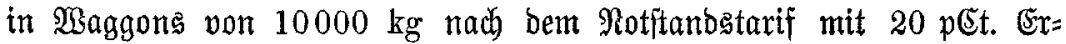

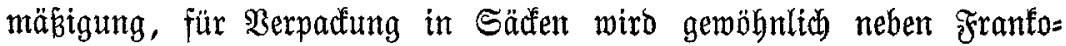
Retournierung berjelben eine Reibgebühr (ca. M 5,- per $\mathfrak{B a g g o n ~ p o n ~}$ $10000 \mathrm{~kg}$ ) bedungen. Doch wirb ber Foritwirt bieje \$erpactung vor= ziehen, ba fie Rabefojten verbilligt, Material ipart, $\mathfrak{A}$ njubr uno $\mathfrak{B e r}=$ teilung weientlich erleidjtert. Bei Berladung in geid)loffenen $\mathfrak{B a g e n}$

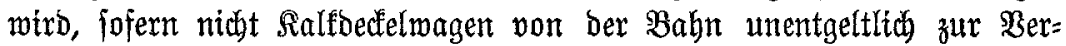
fügung ftehen, ein Frachtzufblag von 10 pet., bet Berlabung in offenen Wagen bie tarifmäß̈ige Dectenmiete erhoben, bie pro Decle $\mathscr{M l} 1,50$ für je $100 \mathrm{~km}$, mindeitens aber $\mathscr{M} 2$,- beträgt. ${ }^{2}$ ) $\mathfrak{U} \mathfrak{m}$ möglichen $\mathfrak{B}$ eiterungen

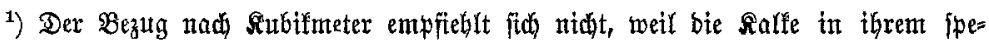

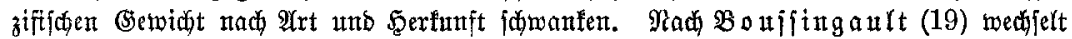

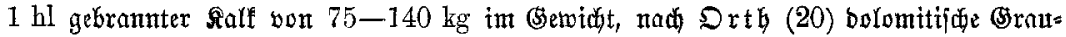

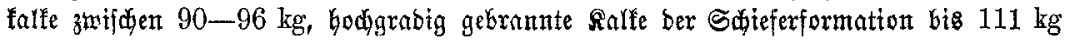
pro Seltoliter.

2) Diefe Hjancen haben jebod nur (seltutg für bent Bereidy ber prentifidien Stants= bahn= Berwaltung uad mir zugegangenen pribaten Mitteilungent. 


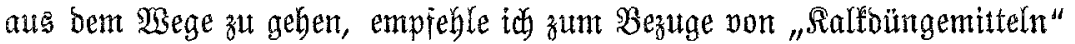

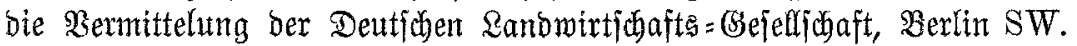
Kodfftr. 73 anzugehen.

\section{Zadhteile.}

Die mannigfachen Schäben, bie bem ßalf für lanbwirtidfaftlidfe

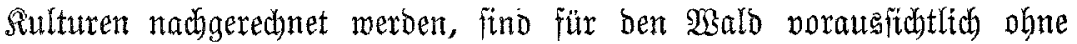
Bebeutung, fie find meittens eine Folgeericheinung von übermäBiger ein= maliger voer zut oft wieberbolter 3ufübrung. Einem Serarmen an Sumnts wirb ber jährlithe \&aubabfall vorbentgen, ein Anäzen ber $\mathfrak{3 u r z e l n , ~}$

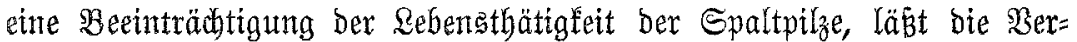
wentoung foblenjauren Ralkes nidht befürchten. Serjagte aber jelbft eine Ralfbüngung für unjeren bejonberen 8 med, jo mird trobbem ber an= ftehenbe ober fommende Beftand von Derielben Muthen zieben. Sicher,

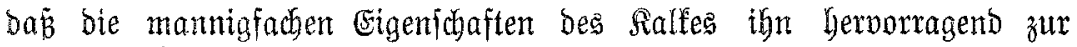

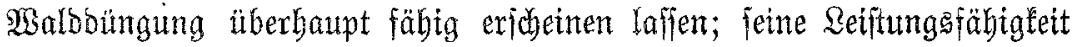
im Speziellen miro nod z̆ erproben fein.

Sind bie in voritebenden 3eilen bargelegten Anjwaumten mun aud meift folche, bie auf ber $\mathfrak{B}$ afte rein lanowirtichaftlicher Praris entitanden

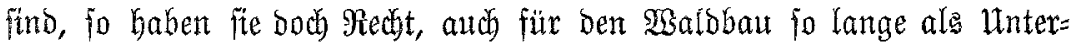
lagen Beartung zu finden, bie fie burch fpeziellere Erfabrungen auth für biejen 2 Sirticuaftszmetg abgelöft werben.

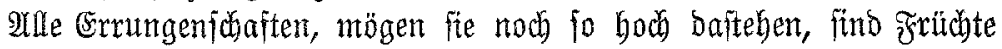

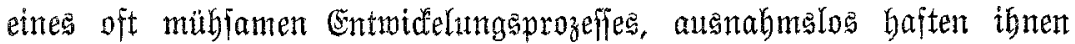
porerit Mängel an, weldhe im saufe ber Beit uns burd bie Erfabrungen Der Zeit jolieblich erfannt, gemilbert, bejeitigt werben, bis ber an und für fich ridftige (Grundgeoante nach und nach feitere Form geminnt.

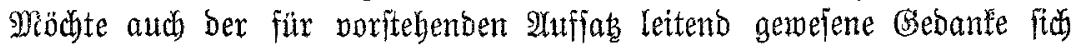
eines 2 Uusbattes fäbig ermeifen!

\section{Bitteratur:}

1. E) bermayer, Bhyftolog. Shemie b. Bflanzen. Berlin 1882, I, S. 790.

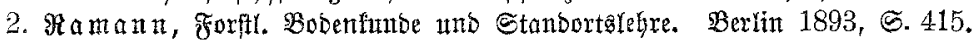

3. Ebernater, loc. cit. ธ. 761 .

4. Braungart, Die $\mathfrak{B i f f e n i \omega a f t ~ i n ~ b e r ~ B o b e n t u n b e . ~ B e r l i n ~ u . ~ R e i p z i g ~ 1 8 7 6 , ~ S . ~} 221$.

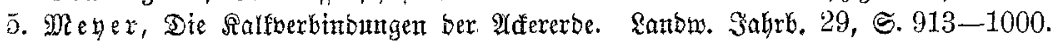

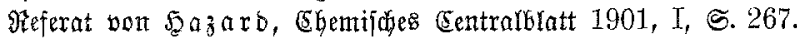

6. Stahl, Bflanzen und Sanedfen. Sena 1888, nnitget, bei Sorth, S. 26.

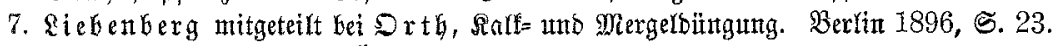

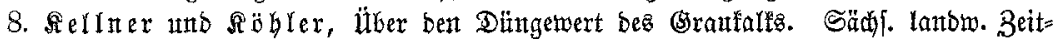

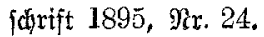

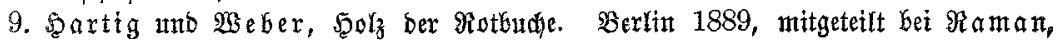
Boberfunde 5. 314 . 
10. Fa man, Forftl. Bobenfunbe mb Standortalehre, S. 415 .

1. Do. Do. bo. S. 316 .

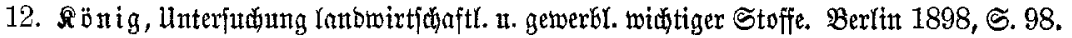

13. Mayer. Die Ditngerlehre in 12 Borlejungen. Sweibelberg 1895, S. 166.

14. $\mathfrak{B}$ itt $\mathfrak{m} \mathfrak{n} \mathfrak{n}$, Der Snaten=, Dìnger $=$ und Fruttermartt. Tir. 49 . Dezentber 1899, ธ. 1153.

15. Mayer, Düngerlehre, S. 98.

16. Drth, 凡alf $=$ tnd Mergelbïngung, S. 141 .

17. van Şermbeef, priwate Mitteilung.

18. Salfelb, mitgetetlt bei Drth; אalf= unb Mergelbüngung, S. 197.

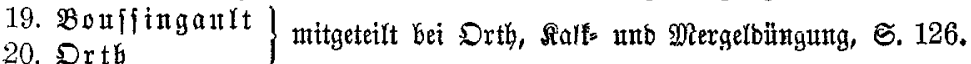

\section{Ütber die Widhtigleit des rajhhen Bodenifhlufjes und der Bejhattung des Bodens bei Kenanpflanjungen auf Kahl: ¡̧lägen.}

Schon an anderer Stelle hatte idh Gelegenheit, auf bie Berjuthe bes

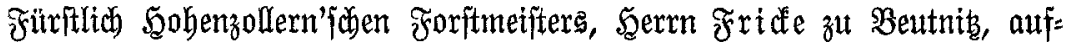

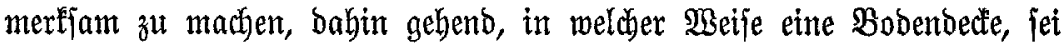
es nun burd bie \$fflanzen felbit ober auch fünftlich gefbaffen, fomohl auf bie Erfhaltung bes Şumus wie auf bas Wadhstum ber Ffflanzen zu

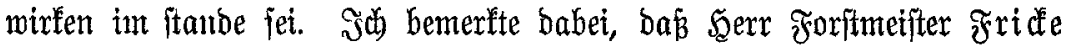
bei 15jäbrigen ßitefern, ca. $50 \mathrm{~cm}$ hoch, Deren Sabrestriebe im Durch)=

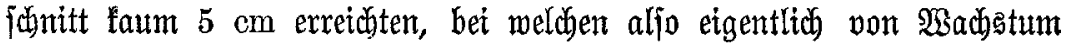
faum bie Rede jein lonnte, verjudte, jowohl burch Dïngung, wie auch

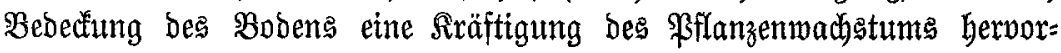
zurufen. Der Boben, um welchen es fich hierbet bandelte, ift ein fait volftändig bumugfreier, fablechter Sanbboben. - ütber bie bisher er= zielten Rejultate liegen mix num ganz genaue Mitteilungen vor, uno ba biejelben von alfiettig gröftem Sntereffe find, namentlich ba, wo es fich um Rulturen auf bürrem, leidbten Sanbboben hanbelt, bringe id fie hier= mit zu allgemeinerer Renntnis.

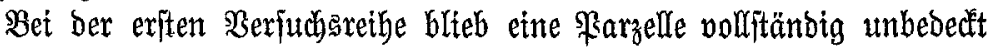
und ungedüngt. Sater erreichten bie Triebe fowohl in ben Safren 1899

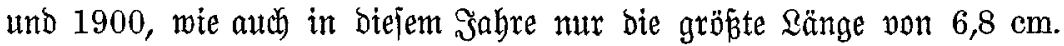

Cine zweite $\mathfrak{s a r z}$ elle wurbe im 2 Uuguft 1898 ftart mit bürrem Rartoffelfraut bebeč̉t, blieb aber ohne jede Düngung. - Dag Rejultat war hier jobon ein recht exfreuliches. Denn nicht mur entwickelten bie sfflanzen bunkelgrüne, geiunde Sabeln, Der Sabrestrieb zeigte aud eine

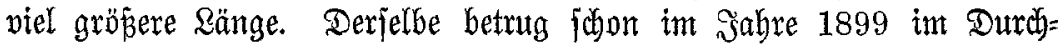

